\title{
A REAL TIME VARIATIONAL DATA ASSIMILATION METHOD BASED ON COMPLEXITY REDUCTION AND ON-THE-FLY DATA DRIVEN EMPIRICAL ENRICHMENT
}

\author{
W. Haik ${ }^{1,2, *}$, Y. Maday ${ }^{1}$ and L. Chamoin ${ }^{2}$ \\ ${ }^{1}$ Sorbonne Université, CNRS, Université de Paris, Laboratoire Jacques-Louis Lions (LJLL) \\ 4, Place Jussieu, 75005 Paris, France \\ e-mail: \{willy.haik,yvon.maday\}@sorbonne-universite.fr \\ ${ }^{2}$ Université Paris-Saclay, ENS Paris-Saclay, CNRS, Laboratoire de Mécanique et Technologie \\ 4, avenue des sciences CS30008, 91192 GIF SUR YVETTE CEDEX \\ e-mail: \{willy.haik,ludovic.chamoin\}@ens-paris-saclay.fr
}

\begin{abstract}
State estimation is a specific data assimilation task in which the quantity of interest is the state of the physical system over a domain of interest. For that purpose, we rely on observations on the system, given by sensors at different sampling times, and a best-knowledge model. However, how good the model may be, it is a deficient representation of the true physics, it may lack some not modeled physics, or parameter values can be inaccurate and erroneous. Therefore, the model bias affects the effectiveness of data assimilation techniques and needs to be corrected with observation data. Moreover, for several years, the explosion of the access to experimental data in industrial systems and the considerable improvements in computing tools have led to the development of data assimilation methods that aim to be used for system monitoring with a real-time constraint. The first idea to decrease the computational cost in the online procedure is to use reduced order modeling (ROM) methods, indeed the reduction of the complexity of the model can overcome the difficulties at the cost of controlling and integrating the model error.
\end{abstract}

The Parameterized Background Data-Weak (PBDW) method introduced in [1] is a non-intrusive, reduced basis, real-time and in-situ data assimilation framework for physical systems modeled by parametrized Partial Differential Equations for steady-state problems. The key idea of the formulation is to seek an approximation to the true field employing projection-by-data, with a first contribution from a deduced background estimate computed from a set of solutions from reduced modeling and a second contribution from an update state informed by the experimental observations (correction of model bias). Further research works [2,3] developed a nonlinear framework to deal with noisy data.

The present study aims at extending the PBDW formulation for time-dependent problems and proposes a sequential version to deal with sampled data. First, a discussion on the integration of time in the reduced order model is studied so that we can apply the PBDW at the current time step. Second, a datadriven empirical enrichment of the model, using a Sequential Karhunen-Loeve algorithm on updates states, is developed in order to learn the not modeled physics of the system and correct the deduced background estimate on-the-fly. Third, a state prediction for future time steps can be computed and integrated in a sequential algorithm with a selection of relevant data, using a parametric identification on the deduced background estimate and an extrapolation of the time function from the tensor-based decomposition (SVD) on prior updates over a window time. Numerical experiments are conducted on a thermal conduction problem in the context of additive manufacturing, with different cases of model bias: a bias on heat source, a biased boundary condition and an error on the constitutive equation. These numerical experiments show that the method significantly reduces the online computational time while providing relevant state evaluations and state predictions, in particular the update plays a significant role in improving the accuracy of the deduced background estimate.

\section{REFERENCES}

[1] Maday, Y., Patera, A. T., Penn, J. D., and Yano, M. (2015). A parameterized background dataweak approach to variational data assimilation: formulation, analysis, and application to acoustics. International Journal for Numerical Methods in Engineering, 102(5), 933-965.

[2] Yvon Maday and Tommaso Taddei (2017) Adaptive pbdw approach to state estimation: noisy observations; user-defined update spaces. arXiv preprint arXiv:1712.09594.

[3] Gong, H., Maday, Y., Mula, O., and Taddei, T. (2019). PBDW method for state estimation: error analysis for noisy data and nonlinear formulation. arXiv preprint arXiv:1906.00810. 